\title{
Benefit function and individual preferences. A generalization of the Zero-Maximum principle
}

\author{
Juan Aparicio* • Jesus Pastor \\ Center of Operations Research (CIO), University Miguel Hernandez of Elche, Spain
}

Received: 12 February 2012

Revised: 18 March 2012

Accepted: 19 March 2012

\begin{abstract}
In this paper we show that given a utility threshold Luenberger's benefit function correctly represents individual preferences as long as specific reference commodity bundles are considered. We further show a condition which is sufficient for reaching Pareto optimality that generalizes the zero-maximum principle proposed by Luenberger. Under our hypothesis, the social benefit could be positive, negative or zero, and not necessarily always zero.
\end{abstract}

Keywords: Benefit function, welfare economics, Pareto optimality, zero-maximum principle

JEL Classification Codes: D00, D60

\section{Introduction}

Twenty years ago, Luenberger (1992a, 1992b) introduced the benefit function. The benefit function, denoted as $b(g ; x, \underline{u})$, measures the amount of a reference bundle $g$ that a generic consumer would be willing to trade for the opportunity to move from a given utility level $\underline{u}$ to a consumption bundle $x^{1}$. The benefit function can be seen as a generalization of the usual willingness-to-pay concept (Luenberger, 1995) and has a number of outstanding properties as we will show later on.

An especially attractive property of the benefit function is that it can be summed up across consumers of an economy to provide a meaningful measure of total benefit, expressed in units of $g$ (Luenberger, 1992b). The importance of this feature lies in the fact that welfare comparisons are usually made at the aggregate level. Along this line,

\footnotetext{
*Corresponding author. E-mail: j.aparicio@umh.es.

Citation: Aparicio, J. and Pastor, J. (2012) Benefit function and individual preferences. A generalization of the Zero-Maximum principle, Economics and Business Letters, 1(1), 12-20.

${ }^{1}$ In order to avoid the possible confusion between the utility level and the utility function, considered subsequently, we denote the former by $\underline{u}$ and the latter by $u($.$) .$
} 
Luenberger (1992b) showed that, under suitable conditions, a Pareto efficient allocation, $X^{*}=\left(x^{1^{*}}, \ldots, x^{n^{*}}\right)$, maximizes the total benefit, $\sum_{i=1}^{n} b_{i}\left(g ; x^{i}, \underline{u}_{i}\right)$, relative to the utility levels $\underline{u}_{i}=u_{i}\left(x^{i *}\right), i=1, \ldots, n$, where $u_{i}\left(x^{i^{*}}\right)$ is the value of the utility function of consumer $i$ at the consumption bundle $x^{i^{*}}$. In this case the total benefit is equal to zero. Luenberger also showed the converse relation: if an allocation $X^{*}=\left(x^{1^{*}}, \ldots, x^{n^{*}}\right)$ maximizes the total benefit relative to $\underline{u}_{i}=u_{i}\left(x^{i *}\right), i=1, \ldots, n$, then the total benefit is zero and $X^{*}=\left(x^{1^{*}}, \ldots, x^{n^{*}}\right)$ is Pareto efficient. In this way the concept of Pareto efficiency is transformed into an optimality principle, called "the zero-maximum principle".

In addition, the benefit function satisfies another interesting property. In particular, Luenberger (1992a) states that the benefit function is "a new representation of individual preferences" and that it is "a new function describing individual preferences" (Luenberger, 1992b). Consequently, the benefit function is a priori particularly well suited for analysing welfare problems.

In this paper, we show that given a utility threshold the benefit function does not always represent individual preferences unless additional conditions are imposed. We have also been able to generalize the relation between Pareto efficiency and optimality of the total benefit function in the following sense: we do not need to assume that the utility levels are determined by the Pareto efficient point and we allow the total benefit to be different from zero. As far as we know there is only one precedent in the literature which attempts to generalize the zero-maximum principle, but assuming core allocations (Courtault et al., 2007).

\section{The benefit function and the individual preferences}

We use the framework of an economy described as follows. There are $m$ commodities and $n$ consumers. Each consumer $i$ has a consumption possibility set $\chi_{i}=R_{+}^{m}$ and a preference ordering described by a continuous and strongly monotonic utility function $u_{i}($.) . Consumers are presumed to be rational and to maximize utility subject to a fixed budget constraint or, equivalently, to minimize expenditure required to achieve a given utility level. We denote the range of values of the function $u_{i}($.$) as U_{i}$. Furthermore, $b_{i}\left(g ; x^{i}, \underline{u}_{i}\right)$ denotes the consumer $i$ 's benefit function given a reference bundle $g$, a consumption bundle $x^{i}$ and a utility level $\underline{u}_{i}$. Additionally, when dealing with a unique consumer, we will not use any subscript, i.e., we will utilize $\chi, u(),$.$U and b(g ; x, \underline{u})$. The precise definition of the benefit function is given below.

\section{Definition 1.}

For any $g, x, \underline{u}$ with $g \in R_{+}^{m}, g \neq 0_{m}, x \in \chi, \underline{u} \in U$ let

$b(g ; x, \underline{u})=\left\{\begin{aligned} & \max \{\beta: x-\beta g \in \chi, u(x-\beta g) \geq \underline{u}\}, \\ & \quad \text { if } x-\beta g \in \chi, \text { and } u(x-\beta g) \geq \underline{u} \text { for some } \beta, \\ &-\infty, \text { otherwise. }\end{aligned}\right.$ 
Geometrically, the benefit function represents the farthest one can move from the consumption bundle $x$ in the direction of $g$ and still achieve the indifference curve associated to $\underline{u}$ (see Figure 1).

Figure 1. The benefit function

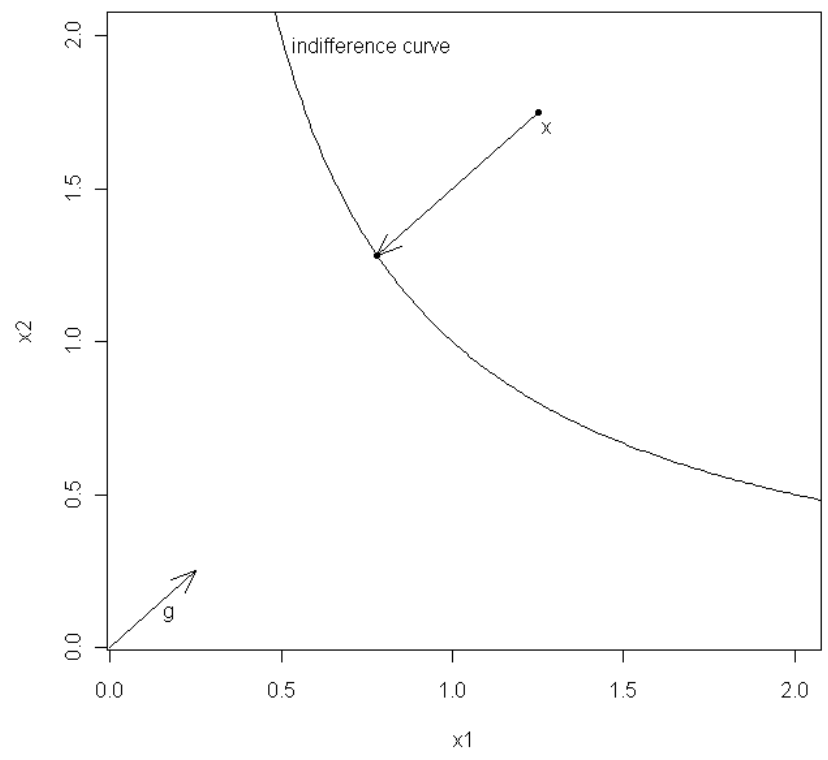

Luenberger (1992a, 1992b) states that the benefit function is a new function describing individual preferences. Next we show a numerical example where, given a utility threshold, the benefit function does not represent the preferences as given by the utility function of an individual. Nevertheless, as a referee points out, it could be understood to mean that the benefit function was not able to describe preferences, and this is not the case. The benefit function is able to describe any indifference curve associated to any utility level and therefore is able to be used to describe preferences. However, the description of the preferences is somehow peculiar in the sense that it is not a monotonic description because larger $b(g ; x, \underline{u})$ does not necessarily correspond to larger utility.

Example 1. Consider $m=2, g=(1,0), \underline{u}=1$ and that the preference relation of an individual can be represented by means of the Cobb-Douglas utility function $u\left(x_{1}, x_{2}\right)=x_{1}^{1 / 2} x_{2}^{1 / 2}$. Now, according to Example 1 of Luenberger (1992a), the individual's benefit function $b(g ; x, 1)$ is equal to $x_{1}-\frac{1}{x_{2}}$. Let us assume that we want to evaluate the value of the bundles $\tilde{x}=(1,9)$ and $\bar{x}=(4,1)$. We get $b_{1}(g ; \tilde{x}, 1)=\frac{8}{9}<$ $b_{1}(g ; \bar{x}, 1)=3$. Therefore, according to the value of the benefit function, it seems that $\bar{x}$ is clearly preferred to $\tilde{x}$. However, using the utility function we get exactly the inverse inequality $u(\tilde{x})=3>u(\bar{x})=2$. 
As a consequence of the above example, we can conclude that the benefit function does not always represent a given individual preference relation. In the next section, we introduce a sufficient condition that guarantees that the preference ordering given by the benefit function is the same as the preference ordering given by the utility function.

\section{The utility translation bundles}

In this section we introduce a new type of bundles that allows us to establish a nice relationship between the utility function and the benefit function.

Definition 2. The non-zero vector $g \in R_{+}^{m}$ is said to be a utility translation bundle if for any $x, x^{\prime} \in \chi$ such that $u(x)=u\left(x^{\prime}\right)$ it holds that $u(x+\alpha g)=u\left(x^{\prime}+\alpha g\right)$ for all $\alpha \in R$.

In other words, if two bundles are indifferent for an individual, the new bundles obtained by adding $\alpha$ times $\mathrm{g}$ are indifferent as well, being $\alpha$ any real constant. It is worth to note that a similar definition has already been deployed in the production theory literature (see the concept of translation homotheticity by Chambers and Färe, 1998).

In what follows, let us assume that for any commodity bundle and for each utility level we can always modify the given commodity bundle, by taking away a certain multiple of the reference bundle, so as to assure that the modified bundle reaches the considered utility level (see Figure 1). Mathematically, for each $x \in \chi$ and for each $\underline{u} \in U$ there exist a $k \in R$ such that $x-k g \in \chi$ and $u(x-k g)=\underline{u}$. Under this assumption, it can be proved that $u(x-b(g ; x, \underline{u}) g)=\underline{u}$.

Proposition 1. Let $x \in \chi, g \in R_{+}^{m}, g \neq 0_{m}$, and $\underline{u} \in U$ such that $\exists k \in R$ with $x-k g \in \chi$ and $u(x-k g)=\underline{u}$. Then $u(x-b(g ; x, \underline{u}) g)=\underline{u}$.

\section{Proof.}

Let us consider $b(g ; x, \underline{u})$ and denote it as $b$. By Definition 1 , it is clear that $b \geq k$ and that $x-b g \in \chi$, and $u(x-b g) \geq \underline{u}$. Moreover, since $x-k g \geq x-b g$, it follows, due to the strong monotonicity of $u($.$) , that u(x-k g) \geq u(x-b g)$, or, equivalently, $\underline{u} \geq u(x-b g)$. Consequently, $\underline{u}=u(x-b g)$.

In order to reach the desired relationship between the utility function and the benefit function we need to introduce the next two lemmas.

Lemma 1. Given $\underline{u} \in U$ and $g$ a utility translation bundle. Let $\tilde{x}, x \in \chi$ such that $u(\tilde{x})=u(x)$. Then $b(g ; \tilde{x}, \underline{u})=b(g ; x, \underline{u})$.

Proof. Let us suppose that $b(g ; \tilde{x}, \underline{u}) \neq b(g ; x, \underline{u})$. Without loss of generality, let us further assume that $b(g ; \tilde{x}, \underline{u})>b(g ; x, \underline{u})$. Consequently, $\tilde{x}-b(g ; x, \underline{u}) g$ $\geq \tilde{x}-b(g ; \tilde{x}, \underline{u}) g$ with at least a strict inequality. Moreover, by strong monotonicity, 
$u(\tilde{x}-b(g ; x, \underline{u}) g)>u(\tilde{x}-b(g ; \tilde{x}, \underline{u}) g)=\underline{u}$. Being g a utility translation bundle, we know that $u(\tilde{x}-b(g ; x, \underline{u}) g)=u(x-b(g ; x, \underline{u}) g)=\underline{u}$, which is a contradiction.

Lemma 2. The benefit function satisfies:

(a) Sign preservation: $u(x) \geq \underline{u}$ implies $b(g ; x, \underline{u}) \geq 0$.

(b) If the utility function is monotonic on $\chi$, then $b(g ; x, \underline{u})$ is monotonic with respect to $x$.

\section{Proof.}

(a) is proved in Luenberger (1992a, p. 464), proposition 2(c).

(b) is proved in Luenberger (1992a, p. 466), proposition 6(a).

Proposition 2. Let us consider a utility translation bundle $g$ and $\bar{u} \in U$. Let $\tilde{x}, x \in \chi$. Then $u(\tilde{x}) \geq u(x) \Leftrightarrow b(g ; \tilde{x}, \underline{u}) \geq b(g ; x, \underline{u})$.

\section{Proof.}

Assume that $u(\tilde{x}) \geq u(x)$. It is clear that $u(\tilde{x}-b(g ; \tilde{x}, u(x)) g)=u(x)$. By Lemma 2(a), $b(g ; \tilde{x}, u(x)) \geq 0$. By Lemma 2(b), $b(g ; \tilde{x}, \underline{u}) \geq b(g ; \tilde{x}-b(g ; \tilde{x}, u(x)) g, \underline{u})$. Finally, applying Lemma $1, b(g ; \tilde{x}-b(g ; \tilde{x}, u(x)) g, \underline{u})=b(g ; x, \underline{u})$. This concludes this part of the proof.

To prove the only if part, we know that $u(\tilde{x}-b(g ; \tilde{x}, \underline{u}) g)=\underline{u}=u(x-b(g ; x, \underline{u}) g)$. This implies, being $g$ a utility translation bundle, that $u(\tilde{x})=u(\tilde{x}+[b(g ; \tilde{x}, \underline{u})-b(g ; \tilde{x}, \underline{u})] g)=u(x+[b(g ; \tilde{x}, \underline{u})-b(g ; x, \underline{u})] g)$. Now, since $x+[b(g ; \tilde{x}, \underline{u})-b(g ; x, \underline{u})] g \geq x$ we have, by strong monotonicity, that $u(x+[b(g ; \tilde{x}, \underline{u})-b(g ; x, \underline{u})] g) \geq u(x)$. Or, equivalently, $u(\tilde{x}) \geq u(x)$.

Proposition 2 states that the preference ordering given by the benefit function matches with the preference ordering given by the utility function, assuming that the reference bundle $g$ is a utility translation bundle. In this manner, Proposition 2 defines the bundles that allow a monotonic representation of the preferences using the benefit function. However, from the proposition it could seem that utility translation bundles exist for any utility function verifying the usual properties, and this is not the case. The existence of utility translation bundles implies that some additional restrictions on the utility function (the translation homotheticity property by Chambers and Färe, 1998), apart from the standard ones, must be verified. Therefore, we want to make clear that this restriction is a "sufficient" condition for the existence of the utility translation bundles. 


\section{The maximum principle}

In this section we study an application to social welfare theory of the benefit function. As already mentioned in the introduction, we are going to generalize the zero-maximum principle proposed by Luenberger (1992b, 1995).

We define an allocation as a collection of $n$ bundles $X=\left(x^{1}, \ldots, x^{n}\right)$ where each $x^{i} \in \chi_{i}, \forall i$. As usual, not all allocations are available in an economy. We say that an allocation is feasible if $\sum_{i=1}^{n} x^{i} \in \Gamma$, where $\Gamma$ represents all available aggregate bundles, and we denote the set of all feasible allocations as $\Upsilon$.

The notion of Pareto efficiency is closely related to optimization. Despite that other authors have shown this relation previously, such as Allais (1943) and Dierker and Lenningaus (1986), Luenberger (1992b) provided a new link between Pareto efficiency and optimality which has a strong economic interpretation. This is a consequence of the introduction of the benefit function which can be summed up across individuals to obtain a total benefit function of social welfare. In particular, Luenberger (1992b) showed that if an allocation $X^{*}$ maximizes the sum of the individual benefits relative to their utility levels $u_{1}\left(x^{1^{*}}\right), \ldots, u_{n}\left(x^{n^{*}}\right)$, then the sum is zero and $X^{*}$ is Pareto efficient, and conversely.

In our own case, getting rid of the condition that the utility levels are $u_{1}\left(x^{1^{*}}\right), \ldots, u_{n}\left(x^{n^{*}}\right)$, the total benefit function may be non-zero at $X^{*}$. Intuitively, it is reasonable that each consumer $i$ establishes a certain "global" utility threshold $\underline{u}_{i}$, valid for any point $x^{i} \in \chi_{i}$. In fact, $\underline{u}_{i}$ represents the minimum utility level that consumer $i$ expects to reach at any optimal allocation. Another alternative interpretation for $\underline{u}_{i}$ is based on its relation with a fixed budget. Each utility threshold can be seen as the level of utility associated to the maximal utility which the consumer $i$ can obtain from a fixed budget constraint and fixed prices of commodities.

Next, we show, assuming that $g$ is a utility translation bundle and considering the vector of utility thresholds, that we can also obtain Pareto efficient allocations by means of the same maximum principle but without requiring that the total benefit is zero.

First we establish a lemma, similar to Lemma 3.2 of Luenberger (1992b).

Lemma 3. Let $\underline{U}=\left(\underline{u}_{1}, \ldots, \underline{u}_{n}\right) \in U_{1} \times \ldots \times U_{n}$ and let $g$ be a utility translation bundle. Suppose that $X^{*}$ is a maximal solution of $B(g ; X, \underline{U}):=\sum_{i=1}^{n} b_{i}\left(g ; x^{i}, \underline{u}_{i}\right)$ over $\Upsilon$. Then, $X^{*}$ is Pareto efficient with respect to the interior of $\Pi=\chi_{1} \times \ldots \times \chi_{n}$.

Proof. Suppose that there is a feasible $X$ in $\operatorname{int}(\Pi)$ with $u_{i}\left(x^{i}\right) \geq u_{i}\left(x^{i^{*}}\right)$ for all $i=1, \ldots, n$ and $u_{t}\left(x^{t}\right)>u_{t}\left(x^{t^{*}}\right)$ for some $t$. Now, by Proposition 2, we have that $b_{i}\left(g ; x^{i}, \underline{u}_{i}\right) \geq b_{i}\left(g ; x^{i *}, \underline{u}_{i}\right) \quad$ and $\quad b_{t}\left(g ; x^{t}, \underline{u}_{t}\right)>b_{t}\left(g ; x^{t^{*}}, \underline{u}_{t}\right)$. As a consequence, $\sum_{i=1}^{n} b_{i}\left(g ; x^{i}, \underline{u}_{i}\right)>\sum_{i=1}^{n} b_{i}\left(g ; x^{i^{*}}, \underline{u}_{i}\right)$, which contradicts that $X^{*}$ is maximal. 
We now state a similar result to Theorem 3.4 in Luenberger (1992b).

Theorem 1. Let $\underline{U}=\left(\underline{u}_{1}, \ldots, \underline{u}_{n}\right) \in U_{1} \times \ldots \times U_{n}$ and let $g$ be a utility translation bundle. Let us assume that $\Gamma$ is convex and that, for each consumer $i, u_{i}$ is strongly quasiconcave. If $X^{*} \in \operatorname{int}(\Pi)$ maximizes $B(g ; X, \underline{U}):=\sum_{i=1}^{n} b_{i}\left(g ; x^{i}, \underline{u}_{i}\right)$ over $\Upsilon$, then $X^{*}$ is Pareto efficient.

Proof. Just rewrite the proof of Theorem 3.4 in Luenberger (1992b, p. 232) using Lemma 3.

We would like to emphasize that in our case, the optimal value of the total benefit can be positive, negative or zero and therefore, it is not necessarily zero.

Example 2. The relation established in Theorem 1 between maximality and Pareto efficiency can be illustrated through an Edgeworth box (Edgeworth, 1932) for the case of distributing a fixed endowment. Suppose that the economy contains only two commodities in amount 10 for both cases. Also there are two individuals, labelled A and B. Then the width and the height of the box, associated to each commodity, is equal to 10. A point inside the box represents a distribution of the two commodities in the following manner. The allocation to $\mathrm{A},\left(x_{1}^{A}, x_{2}^{A}\right)$, is fixed using the lower left-hand corner as the origin while the allocation to individual $\mathrm{B},\left(x_{1}^{B}, x_{2}^{B}\right)$, is determined in a complementary way, by measuring from the upper right-hand corner, i.e., $\left(x_{1}^{B}, x_{2}^{B}\right)=\left(10-x_{1}^{A}, 10-x_{2}^{A}\right)$. We also suppose that $\mathrm{A}$ and $\mathrm{B}$ have the same Leontief utility function $u\left(x_{1}, x_{2}\right)=\min \left\{x_{1}, x_{2}\right\}$. Moreover, let us take $g=(1,1)$. Then it is easy to verify that $g$ is a good reference bundle and, on the other hand, $g$ is also a utility translation bundle ${ }^{2}$.

Under these hypothesis, $b_{i}\left(g ; x^{i}, \underline{u}_{i}\right)=\min \left\{x_{1}^{i}-\underline{u}_{i}, x_{2}^{i}-\underline{u}_{i}\right\}=\min \left\{x_{1}^{i}, x_{2}^{i}\right\}-\underline{u}_{i}, i=\mathrm{A}, \mathrm{B}$ (see Luenberger, 1992a, p. 470). Now, we can try to maximize the total benefit (the sum of the A's benefit and B's benefit). The optimization problem is as follows.

$$
\begin{aligned}
B(g ; X, \underline{u})=\operatorname{Max} & \left\{\min \left\{x_{1}^{A}, x_{2}^{A}\right\}-\underline{u}_{A}+\min \left\{10-x_{1}^{A}, 10-x_{2}^{A}\right\}-\underline{u}_{B}\right\} \\
\text { s.t. } \quad & 0 \leq x_{1}^{A} \leq 10 \\
& 0 \leq x_{2}^{A} \leq 10
\end{aligned}
$$

If we assume that $x_{1}^{A} \leq x_{2}^{A}$ then it is very easy to prove (graphically) that the solution points $\left(x_{1}^{A^{*}}, x_{2}^{A^{*}}\right)$ satisfy $x_{1}^{A^{*}}=x_{2}^{A^{*}}$, with $0 \leq x_{1}^{A^{*}} \leq 10$. We achieve the same result if we assume $x_{1}^{A} \geq x_{2}^{A}$. Therefore, any point $\left(x_{1}^{A^{*}}, x_{2}^{A^{*}}\right)$ with $x_{1}^{A^{*}}=x_{2}^{A^{*}}$ and $0 \leq x_{1}^{A^{*}} \leq 10$ is a solution of the above optimization problem. This set of points has been illustrated in Figure 2a. Thanks to Theorem 1, we are sure that any allocation which lies on that

${ }^{2} u(x+\alpha g)=\min \left\{x_{1}+\alpha, x_{2}+\alpha\right\}=\min \left\{x_{1}, x_{2}\right\}+\alpha$. This implies that the vector $g$ is a utility translation bundle for all the individuals (A and B). 
segment is Pareto efficient. In addition, $B(g ; X, \underline{U})=10-\underline{u}_{A}-\underline{u}_{B}$. This result allow us to show different scenarios where the social benefit is strictly negative (Figure $2 b$ ), zero (Figure 2c) and strictly positive (Figure 2d). Obviously, it depends on the value of the individuals' utility thresholds.

Figure 2. An example of Theorem 1 resorting to a

Leontief utility function for each consumer

(a)

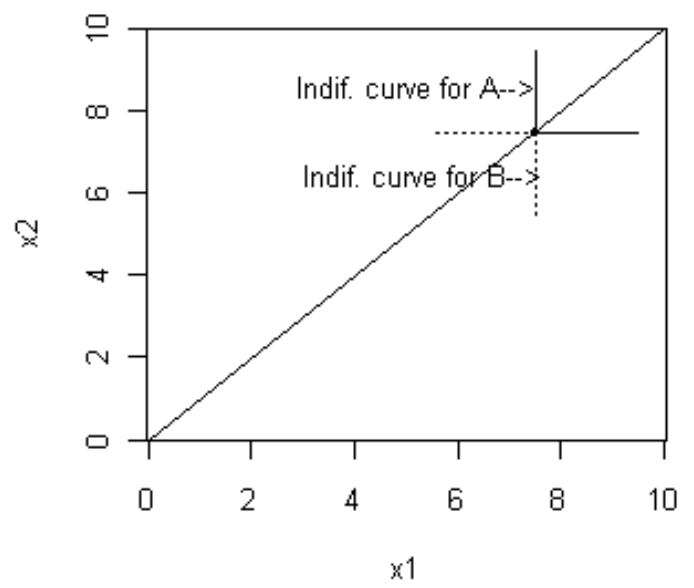

(b)

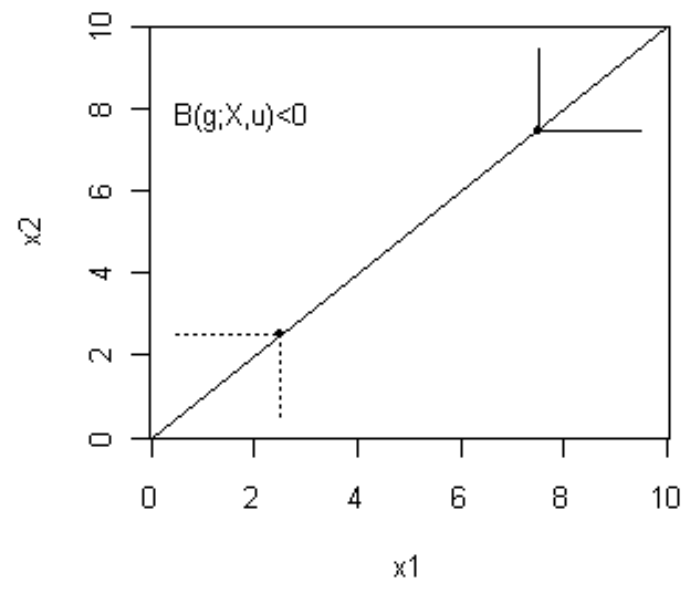

(c)

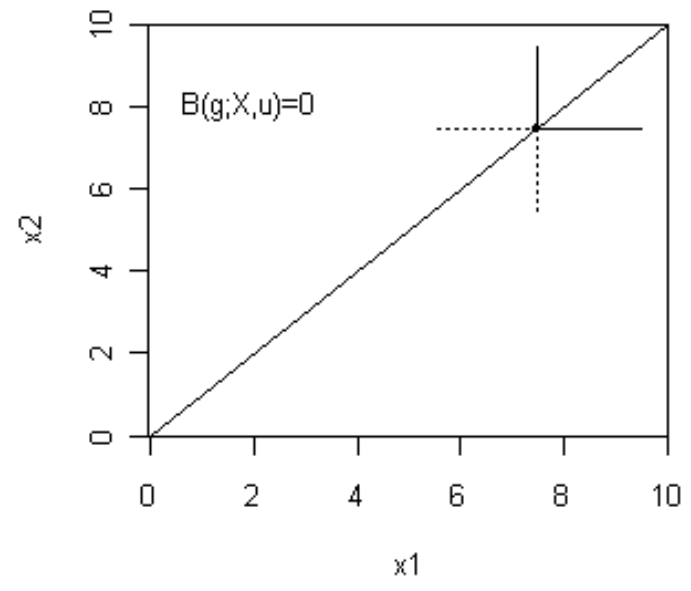

(d)

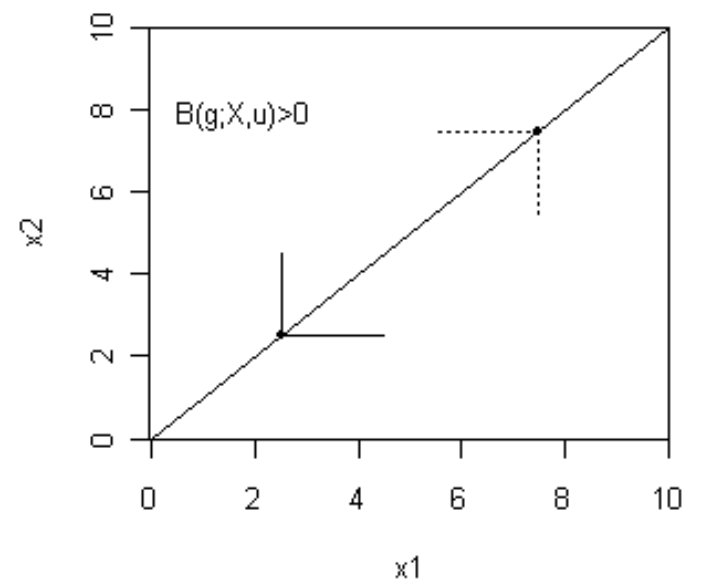

We now turn to the converse of the result stated by means of Theorem 1 . In the case where each consumer $i$ knows that its commodity bundle corresponds to a certain Pareto efficient allocation $X^{*}$, it is reasonable to assume that each consumer will fix its utility threshold as $\underline{u}_{i}=u_{i}\left(x^{i^{*}}\right)$. Consequently, the converse of our Theorem 1 corresponds exactly with Luenberger's Theorem 3.3 (Luenberger, 1992b). 


\section{Conclusions}

In this paper we show, by means of a numerical example, that given a utility threshold Luenberger's benefit function does not always describe correctly individual preferences, as given by the utility function. This has motivated the introduction of a specific type of reference bundles, termed "utility translation bundles", which allows us to assure that the benefit function represents the considered preference ordering.

The same condition allows us to transform the zero-maximum principle of Luenberger into a simple maximum principle in welfare economic theory. It means that the maximum benefit of a whole economy for a given vector of utility thresholds can be positive, negative or zero, and not necessarily always zero.

\section{Acknowledgements}

The authors thank an anonymous referee for providing constructive comments and help in improving the contents and the presentation of this study. They are also grateful to the Ministerio de Ciencia e Innovacion, Spain, and to the Conselleria de Educacion, Generalitat Valenciana, for supporting this research with grants MTM2009-10479 and ACOMP/2011/115, respectively.

\section{References}

Allais, M. (1943) A la Recherche d'une Discipline Economique, 3rd. Ed, (published as Traite d'Economie pure, Clement Juglar, 1994), Paris.

Chambers, R.G. and Färe, R. (1998) Translation homotheticity, Economic Theory, 11, pp. 629-641.

Courtault, J.M., Crettez, B. and Hayek, N. (2007) A Note on Luenberger's ZeroMaximum Principle for Core Allocations, International Game Theory Review, 9(3), 453-460.

Dierker, E. and Lenninghaus, J. (1986) Surplus maximization and pareto-optimality. In Hildenbrand, W. and Mas-Colell, A. (eds.): Contributions to Mathematical Economics, North Holland: Amsterdam, 143-166.

Edgeworth, F.Y. (1932) Mathematical Psychics, Reprint London School of Economics: London.

Luenberger, D.G. (1992a) Benefit functions and duality, Journal of Mathematical Economics, 21, 461-481.

Luenberger, D.G. (1992b) New optimality principles for economic efficiency and equilibrium, Journal of Optimization Theory and Applications, 75(2), 221-264.

Luenberger, D.G. (1995) Microeconomic Theory, McGraw Hill: Boston. 